\title{
Protective Effect of Tert-Butylhydroquinone on the Quinolinic-Acid-Induced Toxicity in Rat Striatal Slices: Role of the Nrf2-Antioxidant Response Element Pathway
}

\author{
Inmaculada Tasset ${ }^{\mathrm{a}}$ Verónica Pérez-De La Cruz ${ }^{\mathrm{b}}$ Diana Elinos-Calderón ${ }^{\mathrm{b}}$ Paul Carrillo-Mora ${ }^{\mathrm{b}}$ \\ Irma Gabriela González-Herrerab ${ }^{b}$ Armando Luna-López ${ }^{d}$ Mina Konigsberg ${ }^{d}$ \\ José Pedraza-Chaverríe Perla D. Maldonadoc Syed F. Alif ${ }^{f} \quad$ Isaac Túnez ${ }^{a, g}$ Abel Santamaríab,f \\ a Departamento de Bioquímica y Biología Molecular, Facultad de Medicina, Universidad de Córdoba, Córdoba, Spain; \\ ${ }^{b}$ Laboratorio de Aminoácidos Excitadores and ' Laboratorio de Patología Vascular Cerebral, Instituto Nacional de \\ Neurología y Neurocirugía Manuel Velasco Suárez, d Departamento de Ciencias de la Salud, División de Ciencias \\ Biológicas y de la Salud, Universidad Autónoma Metropolitana-Iztapalapa, and eDepartamento de Biología, Facultad \\ de Química, Universidad Nacional Autónoma de México, México, D.F., México; ${ }^{f}$ Neurochemistry Laboratory, Division \\ of Neurotoxicology, National Center for Toxicological Research/Food and Drug Administration, Jefferson, Ariz., USA; \\ gInstituto Maimónides de Investigaciones Biomédicas de Córdoba (IMIBIC), Córdoba, Spain
}

\section{Key Words}

Oxidative damage $\cdot$ Quinolinate $\cdot$ Tert-butylhydroquinone $\cdot$

Nrf2 • Antioxidant defense • Glutathione-S-transferase •

Redox signaling

\begin{abstract}
Tert-butylhydroquinone ( $\mathrm{tBHQ}$ ) is a xenobiotic with reported antioxidant properties. $\mathrm{tBHQ}$ has been shown to induce nuclear translocation of the transcription factor NF-E2-related factor 2 ( Nrf2) to further activate the antioxidant response element (ARE). In turn, the Nrf2/ARE pathway is responsible for the induction of phase 2 antioxidant enzymes that detoxify oxidant promoters from different toxic insults. In this work, the antioxidant and protective actions of $\mathrm{tBHQ}$ were explored for the first time on different biomarkers of the neurotoxic model produced by the excitotoxic and pro-oxidant molecule quinolinic acid (QUIN) in rat striatal slices. For comparison purposes, 3-nitropropionic acid was used as reference model. Our results show that tBHQ $(25 \mu \mathrm{M})$ prevented the QUIN-induced lipid peroxidation and mitochondrial dysfunction. In addition, tBHQ enhanced glutathione-S-trans-
\end{abstract}

ferase activity, partially recovering its depletion induced by QUIN treatment. Our results also demonstrated that tBHQ was able to induce nuclear accumulation of Nrf2 and further antioxidant protection: while QUIN alone decreased the nuclear Nrf2, a treatment with $\mathrm{tBHQ}$ preserved the nuclear levels Nrf2 in the presence of QUIN. Therefore, the tBHQ-mediated Nrf2/ARE induction constitutes a signaling-mediated antioxidant strategy and therapeutic tool to be tested in different neurotoxic models.

Copyright $\odot 2009$ S. Karger AG, Basel

\section{Introduction}

Tert-butylhydroquinone (tBHQ), a phenolic compound derived from 3-tert-buthylhydroxyanisol, has been shown to exhibit antioxidant properties $[1,2]$. tBHQ is also able to induce the nuclear translocation of transcription factor NF-E2-related factor 2 (Nrf2), which in turn regulates the expression of vitagenes codifying for cytoprotective phase 2 antioxidant proteins, such as glutathione-S-transferase (GST), NAD(P)H quinone oxidoreduc-

\section{KARGER}

Fax +41613061234 E-Mail karger@karger.ch www.karger.com
(C) 2009 S. Karger AG, Basel

$1424-862 X / 10 / 0181-0024 \$ 26.00 / 0$

Accessible online at:

www.karger.com/nsg
Abel Santamaría

Laboratorio de Aminoácidos Excitadores

Instituto Nacional de Neurología y Neurocirugía Manuel Velasco Suárez

Insurgentes Sur 3877, México, D.F. 14269 (México)

Tel. +52 555606 3822, ext. 2013, E-Mail absada@yahoo.com 
tase, heme-oxygenase 1, $\gamma$-glutamyl-cysteine synthetase, also known as glutamate-cysteine ligase (E.C. 6.3.2.2), and others $[3,4]$. Mostly synthesized in glial cells, Nrf2 is conceptualized as a major antioxidant-driven force in the brain [5]. The expression of antioxidant and phase 2 xenobiotic metabolizing enzyme genes is regulated through cis-acting sequences known as antioxidant response element (ARE). Thus, transcriptional activation through ARE involves Nrf2 nuclear translocation [6].

Evidence from several reports demonstrates that Nrf2 activation represents an accurate strategy to induce astrocyte and neuron cytoprotection through regulation of enzymes belonging to the glutathione redox system [5, $7-11$. Under normal conditions, Nrf2 is sequestered in cytoplasm by its inhibitory protein, Keap-1 [12, 13], and constitutively degraded by proteasome. Reactive oxygen species (ROS) as well as different dietary compounds particularly polyphenols - are able to induce the Keap1/ Nrf2 complex separation, allowing the Nrf2 nuclear translocation $[14,15]$.

The central nervous system is particularly sensitive to the toxic actions of ROS because of: (i) its high oxygen consumption; (ii) its high content in polyunsaturated fatty acids (membrane phospholipids), and (iii) its limited content of antioxidant systems when compared to other tissues [16]. These facts are likely to contribute to the brain oxidative damage observed during the progress of neurodegenerative processes involved in aging and chronic disorders [17-20].

Quinolinic acid (QUIN) is a well-known endogenous tryptophan metabolite, N-methyl-D-aspartate receptor agonist and excitotoxin capable of causing oxidative damage to the mammalian brain. This neurotoxin induces a neurodegenerative pattern in rodents and nonhuman primates that resembles the phenotype and biochemical/ molecular changes in Huntington's disease (HD) [21, 22]. Despite the fact that for a long time the toxic effects of QUIN have been explained through its excitotoxic features, the lack of correlation between the excitotoxic and the neurotoxic effects produced by this agent suggests that additional mechanisms might be involved. In this regard, QUIN-induced oxidative stress directly exerted by $\mathrm{N}$-methyl-D-aspartate receptor overactivation, or independent of this process, represents an alternative explanation to be explored in this paradigm $[23,24]$. In further support of this issue, our group [25] and others [23, 26] have conducted experiments demonstrating that QUIN can be considered a typical pro-oxidant agent in the nervous system.

Nrf2-Mediated Antioxidant Defense
Since most of the antioxidant-based therapies designed at an experimental level to treat neurodegenerative disorders have shown only partial effectiveness, the possibility to target Nrf2 translocation and induce an antioxidant response puts forward a new therapeutic perspective based on transcriptional modulation. Therefore, the purpose of this work was to elucidate whether $\mathrm{tBHQ}$ is capable of reverting the QUIN-induced toxicity and oxidative damage in rat striatal slices by inducing Nrf2 nuclear translocation and activation through ARE expression. For comparative purposes, the toxic model evoked by the mitochondrial toxin 3-nitropropionic acid (3-NPA) was used. Our results not only demonstrated that QUIN exerted a pro-oxidant effect and decreased Nrf2 nuclear levels in the striatal tissue but also that tBHQ treatment counteracted the toxic actions of QUIN and induced a nuclear accumulation of Nrf2, thus suggesting that the Nrf2/ARE pathway accounted for tBHQinduced protection.

\section{Materials and Methods}

\section{Reagents}

QUIN, thiazolyl blue tetrazolium bromide (MTT), malondialdehyde (MDA) and tBHQ were obtained from Sigma Chemical Co. (St. Louis, Mo., USA). All other reagents were from other known commercial sources. Deionized water from a Direct-Q3 UV system (Millipore, Mass., USA) was used for the preparation of solutions.

\section{Animals}

Fifty-eight adult male Wistar bred-in-house rats (250-300 g) were used throughout the study. For all experimental purposes, the animals were housed 5 per cage in polycarbonate box cages and provided with a standard commercial diet (Laboratory rodent diet 5001, PMI Feeds Inc., Richmond, Ind., USA) and water ad libitum. The housing room was maintained under constant conditions of temperature $\left(25 \pm 3^{\circ} \mathrm{C}\right)$, humidity $(50 \pm 10 \%)$ and lighting (12-hour light/dark cycles, light on at 7:00 a.m.). All procedures with animals were strictly carried out according to the National Institutes of Health Guide for the Care and Use of Laboratory Animals and the local guidelines on the ethical use of animals from the Health Ministry of Mexico. During the experiments, all efforts were made to minimize animal suffering.

Preparation and Incubation of Striatal Slices

Striatal slices (200-300 $\mu \mathrm{m}$ of thickness) were obtained fresh from both hemispheres of the rat brains and immediately transferred into an ice-cooled HEPES medium ( $\mathrm{pH}$ 7.4) containing $0.1 \mathrm{M}$ of $\mathrm{NaCl}+0.001 \mathrm{M}$ of $\mathrm{NaH}_{2} \mathrm{PO}_{4}+0.005 \mathrm{M}$ of $\mathrm{NaHCO}_{3}+$ $0.001 \mathrm{M}$ of $\mathrm{CaCl}_{2}+0.006 \mathrm{M}$ of glucose, and $0.01 \mathrm{M}$ of HEPES. Sets of 5 slices per probe were incubated in HEPES medium (final volume of $1 \mathrm{ml}$ ) in a shaking water bath (Dubnoff Lab-Line Incushaker bath) in the presence of tBHQ $(25$ or $50 \mu \mathrm{M})$ for $4 \mathrm{~h}$ at $37^{\circ} \mathrm{C}$. Immediately after incubation with tBHQ, QUIN $(100 \mu \mathrm{M})$ or ve- 
hicle were added and the samples were incubated again at $37^{\circ} \mathrm{C}$ for another $3 \mathrm{~h}$. All the analytical procedures were performed immediately after the last incubation. Six experiments per condition were considered for the statistical analysis.

\section{Lipid Peroxidation Assay}

Lipid peroxidation (LP) was assessed in homogenates obtained from the slices ( 5 slices in a final HEPES volume of $1 \mathrm{ml}$ ) by the assay of thiobarbituric acid-reactive substance (TBA-RS) formation, according to previous reports $[25,27]$. Immediately after incubation, the slices were homogenized, and $250 \mu \mathrm{l}$ of the homogenates were mixed with $500 \mu$ l of the TBA reagent (containing $0.75 \mathrm{~g}$ of TBA $+15 \mathrm{~g}$ of trichloroacetic acid $+2.54 \mathrm{ml}$ of $\mathrm{HCl}$ ), to be reincubated in a boiling water bath $\left(94^{\circ} \mathrm{C}\right)$ for $20 \mathrm{~min}$. Samples were then kept on ice for $5 \mathrm{~min}$ and centrifuged at 3,000 $\mathrm{g}$ for 15 min. The optical density of the supernatants was estimated at a wavelength of $532 \mathrm{~nm}$ in a Genesys 8 spectrophotometer (ColeParmer, Vernon Hills, Ill., USA). The concentrations of MDA (nanomol of MDA per milligram protein) were calculated by interpolation in a standard curve of MDA (constructed in parallel), corrected by the content of protein per sample and expressed as percent of MDA formed versus control.

\section{Functional Assessment of the Slices by the MTT Reduction}

Assay

To further assess the viability of cells from the striatal slices, MTT reduction was measured as a current index of the functional status of the respiratory chain and mitochondrial function, with small modifications to a method previously described [27]. Briefly, after the last incubation, the slices ( 5 per probe) were homogenized, added with $8 \mu \mathrm{l}$ of MTT $(5 \mathrm{mg} / \mathrm{ml})$, and reincubated at $37^{\circ} \mathrm{C}$ for $60 \mathrm{~min}$. The samples were then centrifuged at $15,300 \mathrm{~g}$ for $15 \mathrm{~min}$, and pellets were resuspended in $1 \mathrm{ml}$ of isopropanol. During this first step of centrifugation, supernatants (aqueous phase) were discarded, since it is assumed that most of the mitochondrial fraction responsible for MTT reduction remains in pellets. A second step of centrifugation was then performed at 1,700 $\mathrm{g}$ for $3 \mathrm{~min}$. Quantification of formazan was estimated in supernatants in a Thermospectronic Genesys 8 spectrometer (Cole-Parmer, Vernon Hills, Ill., USA) by measuring optical density at a 570-nm wavelength. The results were expressed as the percentage of MTT reduction with respect to control values. Data from 6 experiments per group were collected and analyzed.

\section{Assessment of GST Activity}

GST (E.C. 2.5.1.18) is a family of enzymes that catalyzes the conjugation of endogenous glutathione with a variety of electrophyllic compounds, and its expression is known to be modulated by Nrf2. In order to evaluate this enzyme activity, we employed the method described by Habig et al. [28], using 1-chloro-2,4-dinitrobenzene as substrate. Optical density was detected at $340 \mathrm{~nm}$ in a Thermospectronic Genesys 8 spectrometer (Cole-Parmer). The results were expressed as enzyme units needed to transform $1 \mu \mathrm{mol}$ of substrate per minute per milligram of protein.

\section{Protein Content Estimation}

For correction of data obtained from the LP and MTT reduction assays, the protein concentration was determined by the method reported by Lowry et al. [29]. The optical density was evaluated in a Thermospectronic Genesys 8 spectrometer (ColeParmer) at $550 \mathrm{~nm}$ wavelength, using bovine serum albumin as standard.

\section{Cytoplasmic and Nuclear Protein Isolation: Subcellular} Fractionation

In order to obtain nuclear and cytoplasmic subcellular fractions, we followed the protocol reported by Sun et al. [30], with some modifications. Briefly, 5 brain slices of each treatment were washed twice with PBS and then placed in a glass Donce homogenizer, with 4 volumes of hypotonic buffer [10 mM HEPES ( $\mathrm{pH}$ 8.0), $10 \mathrm{mM} \mathrm{KCl}, 1.5 \mathrm{mM} \mathrm{MgCl}_{2}, 1 \mathrm{mM}$ DTT, PMSF and protease inhibitor cocktail composed of $1 \mathrm{~mm}$ phenylmethylsulfonyl fluoride, $0.1 \mathrm{~mm}$ pefabloc, $5 \mu \mathrm{g} / \mathrm{ml}$ pepstatin, $5 \mu \mathrm{g} / \mathrm{ml}$ leupeptin and $2 \mu \mathrm{g} / \mathrm{ml}$ aprotinin] and homogenized immediately with $10 \mathrm{up}-$ and-down strokes using a type B pestle, and allowed to swell on ice for $15 \mathrm{~min}$. NP-40 was added to a final concentration of $0.1 \%$ and vortexed vigorously for $15 \mathrm{~s}$, followed by immediate centrifugation for $1 \mathrm{~min}$ at $1,000 \times g$. The supernatant was further purified by centrifugation for $1 \mathrm{~min}$ at $17,000 \times g$ for $20 \mathrm{~min}$ and collected as the cytoplasmic extract. Nuclear extracts were prepared by resuspension of the crude nuclei in an equal volume high-salt buffer (20 mM HEPES, $1.5 \mathrm{mM} \mathrm{MgCl}_{2}, 0.2 \mathrm{mM}$ EDTA, 20\% glycerol, $420 \mathrm{~mm} \mathrm{NaCl}, 1 \mathrm{~mm}$ DTT, $1 \mathrm{mM}$ PMSF and protease inhibitor cocktail) at $4^{\circ} \mathrm{C}$ for $30 \mathrm{~min}$, and the supernatants were collected after centrifugation at $17,900 \times g$ for $5 \mathrm{~min}$.

\section{Immunoblot Analysis}

To concentrate proteins and to eliminate detergent and high salt concentration, the samples were precipitated with $100 \%$ ethanol at $-20^{\circ} \mathrm{C}$ for $1 \mathrm{~h}$ and resuspended in a Tris-HCI pH 6.8 (65 $\mathrm{mM}$ ) buffer. Proteins were quantified by Lowry's method [29] and the equivalent of $60 \mu \mathrm{g}$ of each fraction was diluted in $2 \times$ SDSPAGE sample buffer [125 mM Tris-HCL (pH 6.8), 10\% 2-mercaptoethanol, $4 \%$ sodium dodecyl sulfate, $10 \%$ sucrose and bromophenol blue] and boiled for $5 \mathrm{~min}$, and separated on 10\% SDSPAGE. After electrophoresis, proteins were electrotransferred to Immobilon-P membranes (Millipore). The membranes were blocked with $3 \%$ nonfat milk in TBS-T (50 mM Tris- $\mathrm{HCl}, 150 \mathrm{mM}$ $\mathrm{NaCl}, 0.1 \%$ Tween 20) during $60 \mathrm{~min}$ and incubated with first antibody [Nrf-2 (H-300), Santa Cruz Biotechnology Inc., Santa Cruz, Calif., USA] at $4^{\circ} \mathrm{C}$ over night, washed 3 times with TBS-T and incubated with horseradish-peroxidase-conjugated $\alpha$-mouse IgG secondary antibody (Pierce, Rockford Ill., USA) during 60 min at room temperature. Protein signals were developed with Immobilon Western Chemiluminescent HRP substrate (Millipore). A Ponceau dying was performed in order to ensure homogenity in sample loading. Densitometric analysis was conducted using the ImageJ freeware (NIH).

\section{Statistical Analysis}

All data were expressed as means \pm SEM and analyzed by 1way analysis of variance, followed by Tukey's test for multiple comparisons. Values of $\mathrm{p}<0.05$ were considered of statistical significance. 


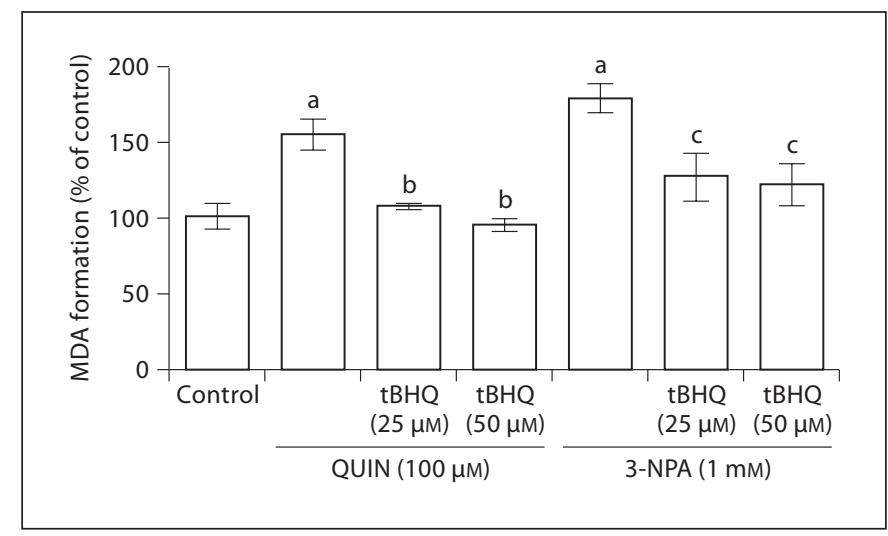

Fig. 1. Effect of tBHQ on QUIN- and 3-NPA-induced MDA formation - as an index of LP - in rat striatal slices. Mean values \pm SEM of 6 experiments per condition. One-way analysis of variance followed by Tukey's test for multiple comparisons. ${ }^{a} \mathrm{p}<$ 0.001: different from control; ${ }^{\mathrm{b}} \mathrm{p}<0.01$ : different from QUIN; ${ }^{\mathrm{c}} \mathrm{p}<0.05$ : different from 3-NPA.

\section{Results}

tBHQ Prevents the QUIN- and 3-NPA-Induced LP

MDA (or TBA-reactive substances) formation was used as LP index in rat striatal slices. The basal LP was $3.9 \mathrm{nmol} \mathrm{MDA} / \mathrm{mg}$ protein (fig. 1). QUIN and 3-NPA treatments significantly enhanced LP by 52 and $75 \%$, respectively. Pretreatment with 25 or $50 \mu \mathrm{M}$ of tBHQ prevented QUIN- and 3-NPA-induced MDA formation. When tested alone at both concentrations, tBHQ did not induce MDA formation (data not shown).

\section{tBHQ Recovered the QUIN- and 3-NPA Decreased \\ Reductive Capacity of Mitochondria}

MTT reduction was employed as an index of the mitochondrial reductive capacity (often interpreted as cell viability) in rat striatal slices. The basal levels of MTT reduction were set as $100 \%$. QUIN and 3-NPA decreased MTT reduction by 40 and 29\%, respectively (fig. 2). Slice preincubation with $25 \mu \mathrm{M}$ of tBHQ resulted in a complete recovery of the reductive mitochondrial capacity in both QUIN and 3-NPA treatments. Interestingly, $50 \mu \mathrm{M} \mathrm{tBHQ}$ was unable to modify QUIN and 3-NPA effects. The fact that only the lower tBHQ concentration tested $(25 \mu \mathrm{M})$ exhibited preventive actions on the mitochondrial MTT reduction capacity suggests that, although the high concentration was still useful against LP (see fig. 1), the same was ineffective to preserve MTT reduction, a relevant issue to consider, since this marker often reflects cell via-

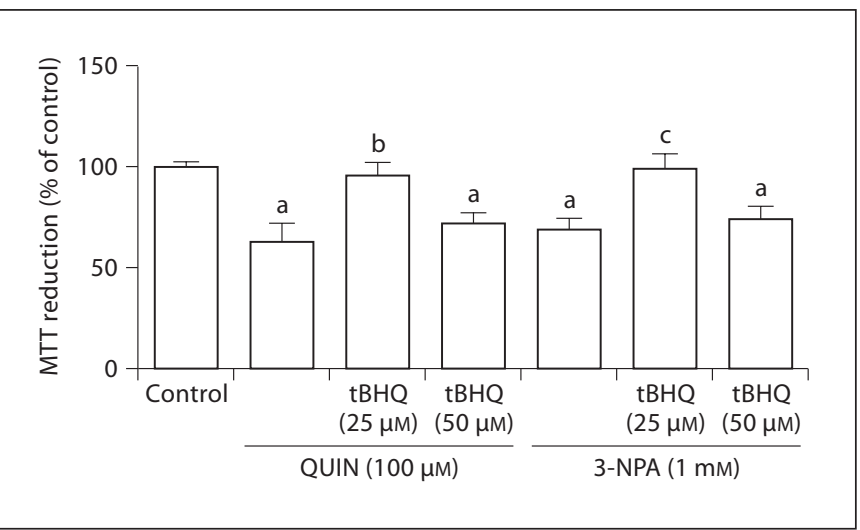

Fig. 2. Effect of tBHQ on QUIN- and 3-NPA-induced decreased MTT reduction - as an index of disrupted mitochondrial reductive capacity - in rat striatal slices. Mean values \pm SEM of 6 experiments per condition. One-way analysis of variance followed by Tukey's test for multiple comparisons. ${ }^{a} \mathrm{p}<0.01$ : different from control; ${ }^{b} \mathrm{p}<0.01$ : different from QUIN; ${ }^{\mathrm{c}} \mathrm{p}<0.05$ : different from 3-NPA.

bility. tBHQ alone had no effect $(25 \mu \mathrm{M})$ or only a moderate decrease in MTT reduction $(50 \mu \mathrm{M})$ as compared to control (data not shown).

\section{tBHQ Induced the Increase in GST Activity}

In order to further investigate whether the protective actions of tBHQ observed in this study were linked to the induction of GST activity, additional experiments were conducted in the striatal slices.

Figure 3a presents the GST activity induced by 25 or $50 \mu \mathrm{M}$ of tBHQ in the slices. Both concentrations were able to enhance this marker similarly (50 and $42 \%$ above the control, respectively) and in a significant manner. This experiment was designed as a first approach to evidence antioxidant responses associated with $\mathrm{tBHQ}$ itself.

When tested in the presence of the toxins (fig. 3b), the GST activity was significantly depleted (53 and 67\% below the control for QUIN and 3-NPA, respectively). Only the lower concentration of tBHQ $(25 \mu \mathrm{M})$ tested was able to induce a partial recovery of GST in both models (35 and $26 \%$ below the control), although these effects were still far of the basal activity levels. Similarly to its action on MTT reduction (see fig. 2), the higher concentration of tBHQ had no preventive effect on GST activity depletion induced in both toxic models, thus suggesting the lower as the optimum concentration of tBHQ to induce protective effects. 

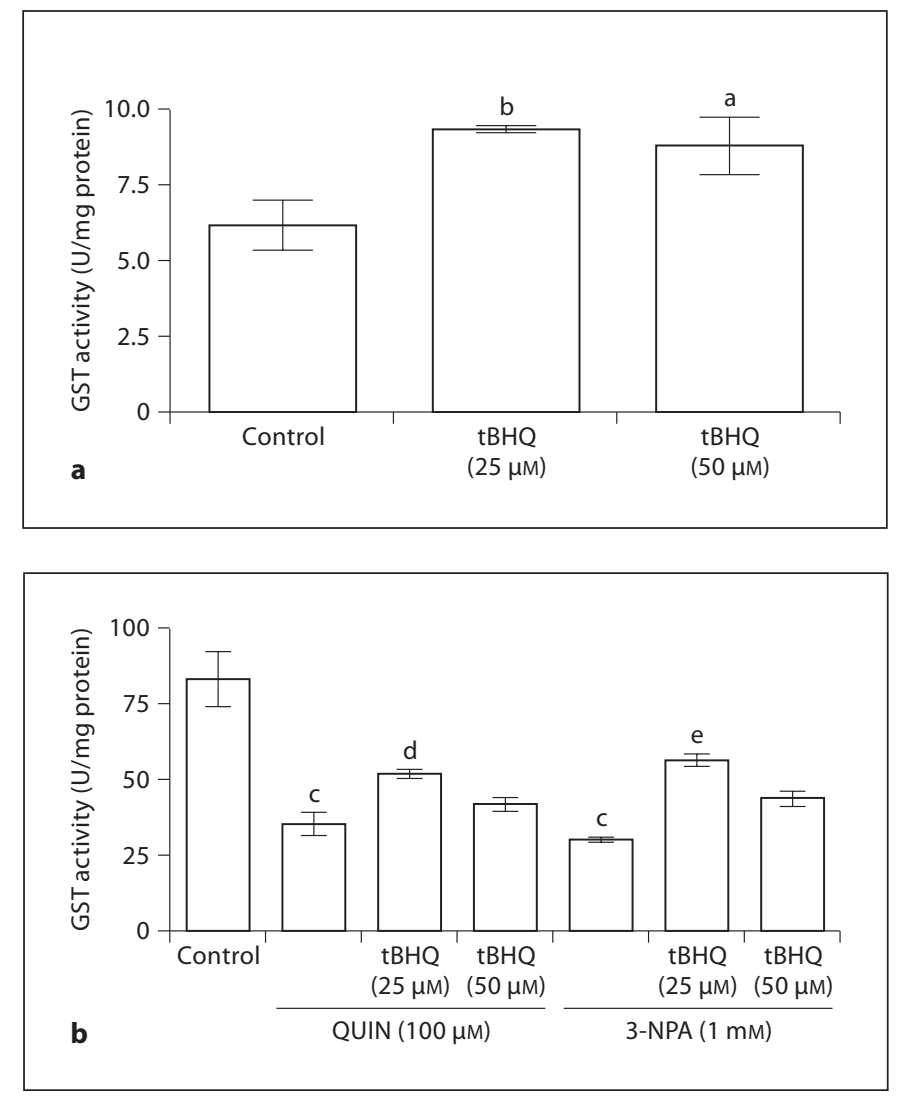

Fig. 3. $\mathrm{tBHQ}$-mediated induction of GST activity. tBHQ was tested alone (a) or in the QUIN and 3-NPA toxic models (b) produced in rat striatal slices. Mean values \pm SEM of 6 experiments per condition. ${ }^{\mathrm{a}} \mathrm{p}<0.05,{ }^{\mathrm{b}} \mathrm{p}<0.01$ : different from control; ${ }^{\mathrm{c}} \mathrm{p}<0.001$ : different from control; ${ }^{\mathrm{d}} \mathrm{p}<0.01$ : different from QUIN; ${ }^{\mathrm{e}} \mathrm{p}<$ 0.001: different from 3-NPA.

The differences in the baselines of GST activities represented in fig. $3 a$ and $b$ are likely to be due to the time of incubation of the slices: in figure $3 \mathrm{a}$ the slices had been incubated only for $4 \mathrm{~h}$, whereas in figure $3 \mathrm{~b}$ the samples had been incubated for $7 \mathrm{~h}$.

\section{Effects of tBHQ and/or QUIN on Nrf2 Nuclear \\ Accumulation}

The levels of Nrf2 protein were determined by Western blot. In basal conditions (C), we detected a strong signal at $57 \mathrm{kDa}$ in nuclear extracts corresponding to Nrf2 (fig. 4). We also found that the treatment with tBHQ produced a significant increase in the Nrf2 protein levels within the nucleus (about 1.5-fold with respect to C), while the QUIN treatment substantially reduced the nuclear Nrf2 protein levels (about 2.6-fold with respect to
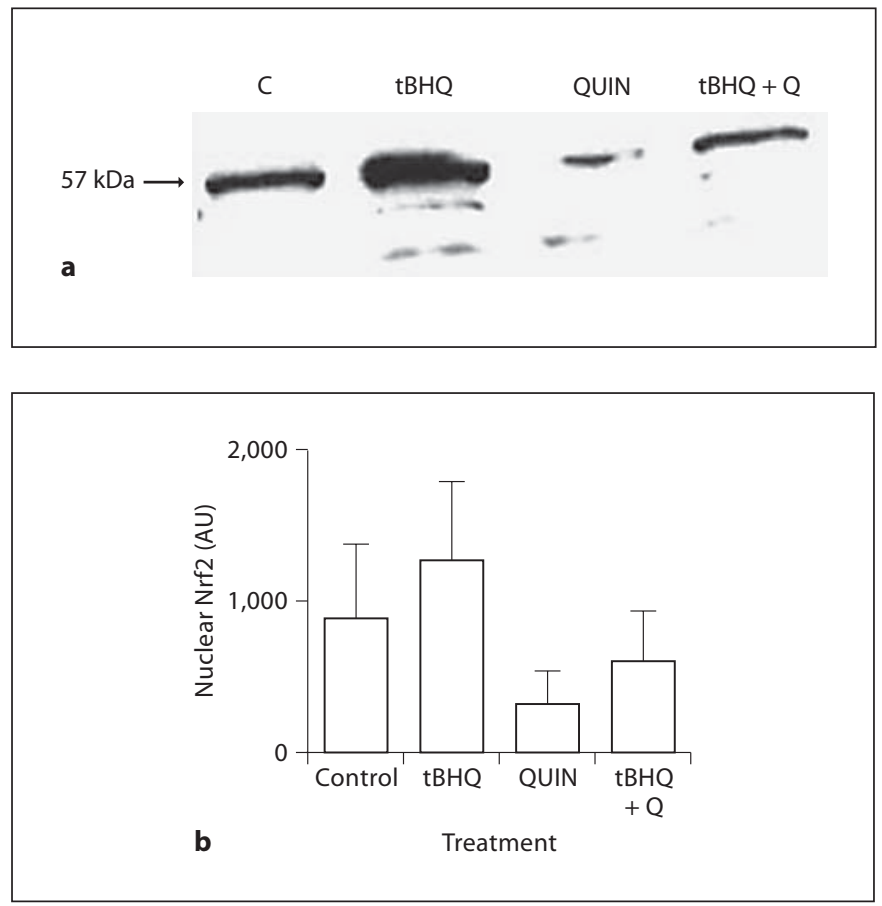

Fig. 4. Immunoblot and densitometric analysis for nuclear Nrf2 protein levels. a Nuclear extract proteins were isolated from rat striatal slices incubated for $4 \mathrm{~h}$ in the absence (c) or presence of tBHQ $(25 \mu \mathrm{M})$ prior to the incubation with QUIN $(100 \mu \mathrm{M})-$ denoted as QUIN and tBHQ + Q treatments. A 57-kDa protein corresponding to Nrf2 signal was seen in the nuclear extracts in all cases. A Ponceau S staining of the nitrocellulose filter showed consistency in the quantity of proteins loaded. Three independent experiments were performed. b Densitometric analysis for all extracts $(n=3$ in each case). Nuclear Nrf2 expression levels are expressed as arbitrary units (AU).

C). In addition, we show that a previous incubation of the slices with tBHQ prior to QUIN treatment reduced the nuclear Nrf2 protein expression with respect to control. In regard to the effect of tBHQ on QUIN-induced Nrf2 depletion, there was a marked increase in the nuclear levels of this factor with respect to QUIN alone (1.4-fold above the control and 1.8-fold above QUIN alone). This result clearly indicates that $\mathrm{tBHQ}$ treatment diminishes the QUIN effect, which in turn might be the consequence of an increased Nrf2 stability mediated by $\mathrm{tBHQ}$, as previously suggested [31]. Notably, in any case, signal was detected at $57 \mathrm{kDa}$ in cytoplasmic extracts (data not shown) in accordance with the findings of Nguyen et al. [31], who also demonstrated in an in vitro study that Nrf2 is primarily a resident of the nucleus. 


\section{Discussion}

Mitochondrial dysfunction, microglial activation, protein aggregates, excitotoxicity and oxidative stress are common features seen in neurodegenerative disorders. Oxidative stress produced by ROS and reactive nitrogen species (RNS) is indeed responsible for the structural and functional alteration in lipids, proteins and DNA. Therefore, the onset and progression of neurodegenerative processes are tightly linked to oxidative damage. Endogenous antioxidant enzymes represent the first line of defense of the brain to counteract the deleterious effects of ROS and RNS, and the expression and activity of these proteins are controlled by ARE, which in turn is activated by Nrf2.

Several recent reports emphasize the importance of the Nrf2 nuclear translocation and further Nrf2/ARE pathway activation as a protective and therapeutic strategy against oxidative stress in in vitro and in vivo models of neurodegenerative disorders [32]. In this study we demonstrated for the first time that the $\mathrm{tBHQ}$-induced Nrf2-associated ARE response readily occurs and accounts for a protective strategy against the pro-oxidant actions of QUIN in an ex vivo model in striatal slices. In addition, QUIN itself produced a depleted expression of nuclear $\mathrm{Nrf} 2$, and this might be suggesting a possible cytoplasmic degradation of this factor by the toxin, which is supported by evidence demonstrating that Nrf2 degradation occurs rapidly in the cytoplasm, thereby accounting for its cytosolic instability [30]. The relevance of these findings is not only inherent in the first characterization of the $\mathrm{tBHQ}$ protective effects in this neurotoxic model (QUIN) through Nrf2 activation but also in the fact that QUIN produces one of the most representative phenotypic models of HD in rodents $[21,33]$. Indeed, QUIN has been shown to be involved in the early stages of HD [22], while it participates in several other neurological disorders. Although the role of Nrf2 induction has already been discussed in other models of HD [5], including the one produced by 3-NPA [34], its description in the QUIN-induced model is particularly important, since this endogenous tryptophan metabolite represents a potential ethiologic factor for the disease.

Several in vitro and in vivo studies have demonstrated that $\mathrm{tBHQ}$ protects neurons from oxidative stress $[35,36]$. This xenobiotic promotes the nuclear translocation of Nrf2, which in turn activates antioxidant enzymes [37], such as GST and $\gamma$-glutamyl-cysteine synthetase. Some groups employing other antioxidant agents, such as nor- dihydroguaiaretic acid, have shown that the stimulation of the Nrf2/ARE pathway involves the induction of hemeoxygenase 1, a major neuroprotective strategy for the preservation of cerebellar granule cells exposed to energy deficit conditions induced by 3 -NPA (the reference model employed by us in this study), or oxidative stress induced by hydrogen peroxide [34]. Our results suggest that tBHQ-induced Nrf2 recruitment may be a protective strategy against oxidative damage also occurring in a model (QUIN) typically linked to excitotoxicity and oxidative damage [33].

Similar to HD, the QUIN model is characterized by excitotoxic events, as well as intense oxidative damage accompanied by high levels of products of lipid oxidation, mitochondrial dysfuntion, etc. [38]. The data collected from this study also show that QUIN depleted the nuclear translocation of Nrf2, a mechanism that might be accounting for its general toxic pattern. In turn, this may also help explain why the biochemical analysis of GST activity and mitochondrial function are both negatively affected by the toxin. In contrast, other studies demonstrate that 3-NPA, the micotoxin producing another phenotypic model of $\mathrm{HD}$, is responsible for inducing biochemical and molecular alterations ultimately leading to Nrf2 translocation [35], although it is necessary to emphasize that this is a mechanistically different model. Moreover, in spite of the fact that Nrf2 is a transcription factor that has been shown to induce ARE by promoting vitagenes that codify for antioxidant proteins [15], it seems that for the QUIN toxic model, the decreased induction of the Nrf2/ARE pathway signaling might be leading to oxidative damage probably via the GST inactivation.

In contrast, tBHQ readily exerts a considerable efficacy in the antioxidant response triggered by $\mathrm{Nrf} 2$ in the striatal slices, which was evidenced by the prevention and protection of those biomarkers of oxidative damage and mitochondrial dysfunction, as well as on the induction of activity of the phase 2 antioxidant enzyme GST, thus supporting the general antioxidant profile already reported for tBHQ [2].

Our data also revealed that the coincubation of the slices with $\mathrm{tBHQ}+\mathrm{QUIN}$, resulting in a significant amelioration of the QUIN-induced LP and MTT reduction, can be linked with the Nrf2 nuclear accumulation seen in this experimental condition, as the nuclear levels of Nrf2 were partially preserved (see fig. 4). Furthermore, while in other toxic models there is evidence suggesting that Nrf2 translocation may also occur as a compensatory response to the action of the ROS/RNS produced 
[15], this is apparently not occurring in this model, although we cannot discard the possibility that at earlier times, the same compensatory response might be taking place. Nonetheless, the effect evoked by tBHQ on Nrf2 may also be following a different pathway than that induced by ROS, probably involving kinase-related cascades [39], or even acting as a real scavenger on the ROS/ RNS generated by QUIN, being oxidized before triggering its effect on the Keap1-Nrf2 complex.

A final issue to consider is whether the tBHQ- and QUIN-induced responses that are associated with the Nrf2/ARE pathway are dependent or not on the NMDA receptor function, given that QUIN is known to possess the ability to stimulate these receptors [21], and so, part of its toxic pattern is exerted via excitotoxicity. This question remains to be investigated in a future study. In the meantime, the induction of the Nrf2/ARE pathway could represent a major explanation for the protective actions that other antioxidants have been reported to exert in the toxic model of QUIN, both under in vitro and in vivo conditions $[33,38,40]$. Whether this pathway is also activated by some of these agents is an issue that will be elucidated by us. Most importantly, our data also provide molecular support to the hypothesis that our group and others have postulated for a long time: oxidative stress signaling represents an alternative nonexcluding explanation for the toxic pattern exerted by QUIN in the brain.

\section{Conclusion}

Findings of this work can be summarized as follows: (i) QUIN produces oxidative stress and mitochondrial dysfunction in rat strital slices, while it inhibits Nrf2 nuclear translocation; (ii) tBHQ acts as an inducer of antioxidant responses through the induction of the Nrf2/ ARE pathway, thus providing preventive and protective actions against the toxic events elicited by QUIN and 3NPA (as a reference toxic model). The proved induction of the Nrf2/ARE pathway provides additional support to the actions of antioxidant agents previously tested in this neurotoxic model, since it brings evidence of protective mechanisms based on molecular adaptations. Although tBHQ seems to be a potential therapeutic tool to be tested in this and other models, more detailed studies are required in order to clarify the precise role that Nrf2 plays in neurodegenerative disorders and whether it may constitute a target for the design of therapeutic strategies.

\section{Acknowledgements}

This work was supported by CONACyT-Mexico Grant 48370Q (A.S.) and DGAPA PAPIIT (IN 207007) (J.P.).

\section{References}

1 Gharavi N, Haggarty S, El-Kadi AO: Chemoprotective and carcinogenic effects of tertbutylhydroquinone and its metabolites. Curr Drug Metab 2007;8:1-7.

-2 Nakamura Y, Kumagai T, Yoshida C, Naito Y, Miyamoto M, Ohigashi H, Osawa T, Uchida K: Pivotal role of electrophilicity in glutathione S-transferase induction by tert-butylhydroquinone. Biochemistry 2003; 42: 4300-4309.

- 3 Talalay P, Dinkova-Kostova AT, Holtzclaw WD: Importance of phase 2 gene regulation in protection against electrophile and reactive oxygen toxicity and carcinogenesis. Adv Enzyme Regul 2003;43:121-134.

-4 Kong AN, Owuor E, Yu R, Hebbar V, Chen C, Hu R, Mandlekar S: Induction of xenobiotic enzymes by the MAP kinase pathway and the antioxidant or electrophile response element (ARE/EpRE). Drug Metab Rev 2001; $33: 255-271$

-5 Johnson JA, Johnson DA, Kraft AD, Calkins MJ, Jakel RJ, Vargas MR, Chen PC: The Nrf2ARE pathway: an indicator and modulator of oxidative stress in neurodegeneration. Ann NY Acad Sci 2008;1147:61-69.

6 Wang W, Chan JY: Nrf1 is targeted to the endoplasmic reticulum membrane by an $\mathrm{N}$-terminal transmembrane domain. J Biol Chem 2006;281:19676-19687.

7 Dringen R, Gutterer JM, Hirrlinger J: Glutathione metabolism in brain metabolic interaction between astrocytes and neurons in the defense against reactive oxygen species. Eur J Biochem 2000;267:4912-4916.

$\checkmark 8$ Shih AY, Johnson DA, Wong G, Kraft AD, Jiang L, Erb H, Johnson JA, Murphy TH: Coordinate regulation of glutathione biosynthesis and release by Nrf2-expressing glia potently protects neurons from oxidative stress. J Neurosci 2003;23:3394-3406.

-9 Kraft AD, Johnson DA, Johnson JA: Nuclear factor E2-related factor 2-dependent antioxidant response element activation by tert-butylhydroquinone and sulforaphane occurring preferentially in astrocytes conditions neurons against oxidative insult. J Neurosci 2004;24:1101-1112.
10 Vargas MR, Johnson DA, Sirkis DW, Messing A, Johnson JA: Nrf2 activation in astrocytes protects against neurodegeneration in mouse models of familial amyotrophic lateral sclerosis. J Neurosci 2008;28:1357413581

-11 Harvey CJ, Thimmulappa RK, Singh A, Blake DJ, Ling G, Wakabayashi N, Fujii J, Myers A, Biswal S: Nrf2-regulated glutathione recycling independent of biosynthesis is critical for cell survival during oxidative stress. Free Radic Biol Med 2009;46:443-453.

-12 Itoh K, Wakabayashi N, Katoh Y, Ishii T, Igarashi K, Engel JD, Yamamoto M: Keap1 represses nuclear activation of antioxidant responsive elements by Nrf2 through binding to the amino-terminal Neh2 domain. Genes Dev 1999;13:76-86.

13 Kang MI, Kobayashi A, Wakabayashi N, Kim SG, Yamamoto M: Scaffolding of Keap1 to the actin cytoskeleton controls the function of Nrf2 as key regulator of cytoprotective phase 2 genes. Proc Natl Acad Sci USA 2004;101:2046-2051. 
-14 Calabrese V, Ravgana A, Colombrita C, Scapagnini G, Guagliano E, Calvani M, Butterfield DA, Giuffrida-Stella AM: Acetylcarnitine induces heme oxygenase in rat astrocytes and protects against oxidative stress: involvement of the transcription factor $\mathrm{Nrf} 2$. J Neurosci Res 2005;79:509-521.

-15 Calabrese V, Cornelius C, Mancuso C, Pennisi G, Calafato S, Bellia F, Bates TE, Giuffrida Stella AM, Schapira T, Dinkova Kostova AT, RizzarelliE: Cellular stress response: a novel target for chemoprevention and nutritional neuroprotection in aging, neurodegenerative disorders and longevity. Neurochem Res 2008;33:2444-2471.

16 Von Bernhardi R: Aging: biochemistry and functional changes of the central nervous system. Rev Chil Neuro-Psiquiatr 2005;43: 297-304.

17 Dröge W, Schipper HM: Oxidative stress and aberrant signaling in aging and cognitive decline. Aging Cell 2007;6:361-370.

18 Halliwell B: Oxidative stress and neurodegeneration: where are we now? J Neurochem 2006;97:1634-1658.

19 Moreira PI, Siedlak SL, Aliev G, Zhu X, Cash AD, Smith MA, Perry G: Oxidative stress mechanisms and potential therapeutics in Alzheimer disease. J Neural Transm 2005; 112:921-932.

$\checkmark 20$ Beal MF: Oxidatively modified proteins in aging and disease. Free Radic Biol Med 2002; 32:797-803.

-21 Stone TW: Neuropharmacology of quinolinic and kynurenic acids. Pharmacol Rev 1993; 45:309-379.

-22 Guidetti P, Luthi-Carter RE, Augood SJ, Schwarcz R: Neostriatal and cortical quinolinate levels are increased in early grade Huntington's disease. Neurobiol Dis 2004; 17:455-461

-23 Behan WM, McDonald M, Darlington LG, Stone TW: Oxidative stress as a mechanism for quinolinic acid-induced hippocampal damage: protection by melatonin and deprenyl. Br J Pharmacol 1999;128:1754-1760.

-24 Santamaría A, Jiménez-Capdeville ME, Camacho A, Rodriguez-Martínez E, Flores A, Galván-Arzate S: In vivo hydroxyl radical formation after quinolinic acid infusion into rat corpus striatum. Neuroreport 2001;12: 2693-2696
25 Santamaría D, Espinoza-González V, Ríos C, Santamaría A: N-omega-nitro-L-arginine, a nitric oxide synthase inhibitor, antagonizes quinolinic acid-induced neurotoxicity and oxidative stress in rat striatal slices. Neurochem Res 1999;24:843-848.

26 Rossato JI, Ketzer LA, Centurião FB, Silva SJ, Lüdtke DS, Zeni G, Braga AL, Rubin MA, Rocha JB: Antioxidant properties of new chalcogenides against lipid peroxidation in rat brain. Neurochem Res 2002;27:297-303.

27 Pérez-De La Cruz V, González-Cortés C, Pedraza-Chaverrí J, Maldonado PD, AndrésMartínez L, Santamaría A: Protective effect of S-allylcysteine on 3-nitropropionic acidinduced lipid peroxidation and mitochondrial dysfunction in rat brain synaptosomes Brain Res Bull 2006;68:379-383.

28 Habig WH, Pabst MJ, Jakoby WB: Glutathione S-transferases: the first enzymatic step in mercapturic acid formation. J Biol Chem 1974;249:7130-7139.

29 Lowry OH, Rosebrough NJ, Farr AL, Randall RJ: Protein measurement with the folin phenol reagent. J Biol Chem 1951;193:265275.

30 Sun Z, Zhang S, Chan JY, Zhang DD: Keap1 controls postinduction repression of the Nrf2-mediated antioxidant response by escorting nuclear export of Nrf2. Mol Cell Biol 2007;27:6334-6349.

-31 Nguyen T, Sherratt PJ, Nioi P, Yang CS, Pickett CB: Constitutive and inducible expression of ARE-driven genes through a dynamic pathway involving nucleocytoplasmic shuttling by Keap1. J Biol Chem 2005;280: 32485-32492.

32 De Vries HE, Witte M, Hondius D, Rozemuller AJM, Drukarch B, Hoozemans J, van Horssen J: Nrf2-induced antioxidant protection: a promising target to counteract ROS-mediated damage in neurodegenerative disease? Free Radic Biol Med 2008;45: 1375-1383.

33 Silva-Adaya D, Pérez-De La Cruz V, HerreraMundo N, Mendoza-Macedo K, VilledaHernández J, Binienda Z, Ali SF, Santamaría A: Excitotoxic damage, disrupted energy metabolism, and oxidative stress in the rat brain: antioxidant and neuroprotective effects of L-carnitine. J Neurochem 2008;105: 677-689.
34 Guzmán-Beltrán S, Espada S, Orozco-Ibarra M, Pedraza-Chaverrí J, Cuadrado J: Nordihydroguaiaretic acid activates the antioxidant pathway Nrf2/HO-1 and protects cerebellar granule neurons against oxidative stress. Neurosci Lett 2008;447:167-171.

35 Shih AY, Imbeault S, Barakauskas V, Erb H, Jiang L, Li P, Murphy T: Induction of the Nrf2-driven antioxidant response confers neuroprotection during mitochondrial stress in vivo. J Biol Chem 2005;280:2292522936.

36 Zhao J, Moore AN, Redell JB, Dash PK: Enhancing expression of Nrf2-driven genes protects the blood brain barrier after brain injury. J Neurosci 2007;27:10240-10248.

>37 Wierinckx A, Brevé J, Mercier D, Schultzberg M, Drukarch B, Van Dam AM: Detoxification enzyme inducers modify cytokine production in rat mixed glial cells. J Neuroimmunol 2005; 166:132-143.

-38 Pérez-De La Cruz V, González-Cortés C, Galván-Arzate S, Medina-Campos ON, Pérez-Severiano F, Ali SF, Pedraza-Chaverrí J, Santamaría A: Excitotoxic brain damage involves early peroxynitrite formation in a model of Huntington's disease in rats: protective role of iron porphyrinate 5,10,15,20tetrakis (4-sulfonatophenyl) porphyrinate iron (III). Neuroscience 2005;135:463-474.

-39 Surh YJ, Kundu JK, Na HK: Nrf2 as a master redox switch in turning on the cellular signaling involved in the induction of cytoprotective genes by some chemopreventive phytochemicals. Planta Med 2008;74:15261539.

40 Pérez-Severiano F, Rodríguez-Pérez M, Pedraza-Chaverrí J, Maldonado PD, MedinaCampos ON, Ortíz-Plata A, Sánchez-García A, Villeda-Hernández J, Galván-Arzate S, Aguilera P, Santamaría A: S-Allylcysteine, a garlic-derived antioxidant, ameliorates quinolinic acid-induced neurotoxicity and oxidative damage in rats. Neurochem Int 2004;45:1175-1183 